\title{
Multiterminal Josephson Effect
}

\author{
Natalia Pankratova, ${ }^{1, *}$ Hanho Lee,,${ }^{1, *}$ Roman Kuzmin, ${ }^{1}$ Kaushini Wickramasinghe, ${ }^{1,2}$ William Mayer, ${ }^{2}$ \\ Joseph Yuan, ${ }^{2}$ Maxim G. Vavilov, ${ }^{3}$ Javad Shabani, $^{2}$ and Vladimir E. Manucharyan ${ }^{1}$ \\ ${ }^{1}$ Department of Physics, Joint Quantum Institute, and Quantum Materials Center, \\ University of Maryland, College Park, Maryland 20742, USA \\ ${ }^{2}$ Center for Quantum Phenomena, Department of Physics, New York University, New York 10003, USA \\ ${ }^{3}$ Department of Physics, University of Wisconsin-Madison, Madison, Wisconsin 53706, USA
}

(Received 19 January 2019; accepted 13 July 2020; published 2 September 2020; corrected 27 October 2020)

\begin{abstract}
We report a probable observation of the dc Josephson effect in mesoscopic junctions of three and four superconductors. The devices are fabricated in a top-down fashion from a hybrid semiconductorsuperconductor InAs/Al epitaxial heterostructure. In general, the critical current of an $N$-terminal junction is an $(N-1)$-dimensional hypersurface in the space of bias currents, which can be reduced to a set of critical current contours. The geometry of critical current contours exhibits nontrivial responses to electrical gating, magnetic field, and phase bias, and it can be reproduced by the scattering formulation of the Josephson effect generalized to the case of $N>2$. Besides establishing solid ground beneath a host of recent theory proposals, our experiment accomplishes an important step toward creating trijunctions of topological superconductors, essential for braiding operations.
\end{abstract}

DOI: 10.1103/PhysRevX.10.031051

Subject Areas: Condensed Matter Physics Mesoscopics, Superconductivity

\section{INTRODUCTION}

A multiterminal Josephson junction [1] consists of $N>2$ independent superconductors (terminals) coupled to each other through a normal scattering region [Fig. 1(a)]. In this case, the supercurrent $I_{j}$ in terminal $j$ becomes a multivariable function of all the $N-1$ independent phase differences $\phi_{j}-\phi_{N}$ (the $N$ th terminal can be assumed grounded). In theory, such a nonlocal coupling of supercurrents originates from the formation of Andreev bound states (ABSs) [2], the wave functions of which are nearly equally present in all of the terminals. Multiterminal Josephson junctions were conceived decades ago in the context of supercurrent transistors [3], direct-current flux transformers [4,5], and superconducting qubits [6].

Recently, the physics of multiterminal ABSs became the subject of numerous theoretical proposals [7-36]. The first category of proposals explores the band topology of multiterminal Josephson junctions [7-21]. These papers explore the structure of the ABS energies in the $(N-1)$ dimensional Brillouin zone, where the phase differences $\phi_{j}-\phi_{N}$ play a role of quasimomenta. Theoretical studies

\footnotetext{
*These authors contributed equally to this work.
}

Published by the American Physical Society under the terms of the Creative Commons Attribution 4.0 International license. Further distribution of this work must maintain attribution to the author(s) and the published article's title, journal citation, and DOI. of such multiterminal junctions predicted band structures with topologically protected states [9-13] and Weyl nodes [14-17]. In the second category of theoretical proposals, a junction of more than two topological superconductors is used to perform braiding operations on the zero-energy Majorana bound states [22-36], as these operations are essential for realization of topologically protected quantum computation [37]. The demonstration of coherent transport in multiterminal Josephson junctions is an important stepping stone for bringing these numerous theoretical proposals to fruition.

On the experimental side, although nanowire network systems proximitized by superconductors have been investigated as a promising platform for topological quantum computation, the experiments are still limited to measuring two-terminal properties [38-43]. Multiterminal supercurrents were reported in graphene-based junctions [44], where a heating effect of coexisting disspative currents on supercurrent transport was mainly discussed. Other relevant experimental developments include the observations of the out-of-equilibrium Cooper pair quartet transport in superconducting bijunctions [45-47] and the phase-controlled superconducting proximity effect in metallic three-terminal junctions [48]. Mesoscopic junctions exhibiting superconducting phase coherence simultaneously between multiple terminals remained elusive, which nonetheless is the key property required in both braiding and topological band structure proposals.

The peculiarity of Josephson junctions with $N>2$ can be appreciated already while defining the key notion of the 
(a)

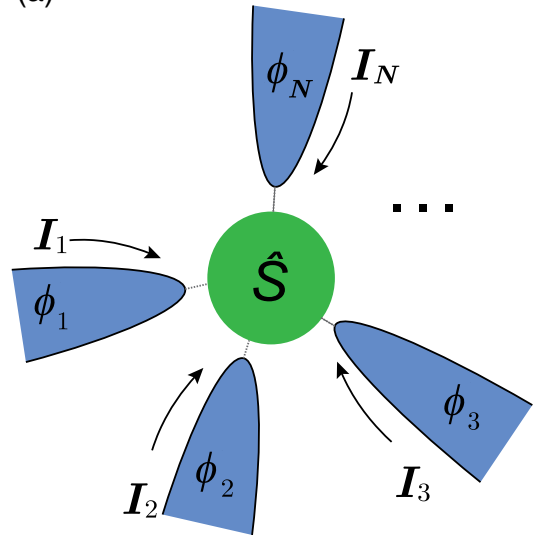

(b)

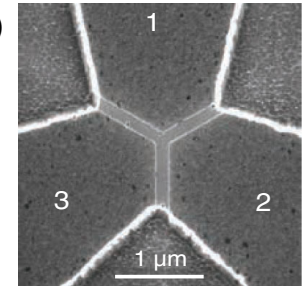

(d)

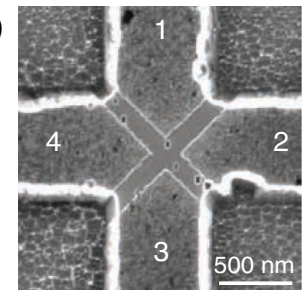

(c)

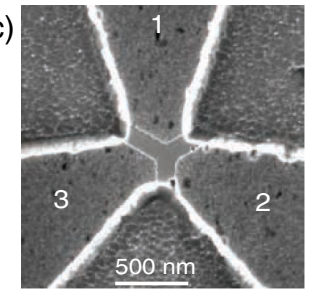

(e)

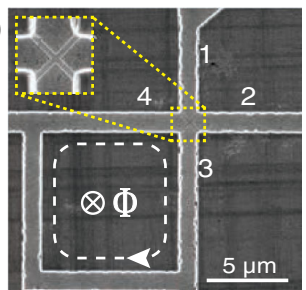

(f)
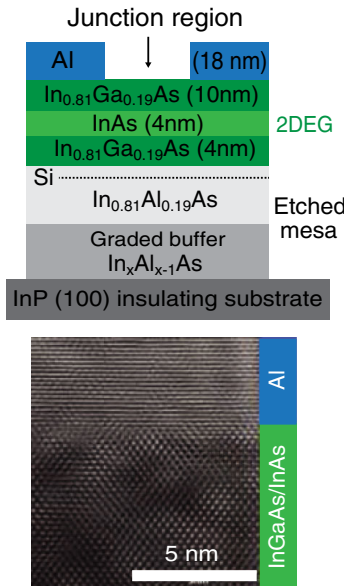

FIG. 1. Multiterminal Josephson junction. (a) A junction of $N$ superconducting terminals (blue) across a normal scattering region (green), which is characterized by the scattering matrix $\hat{S}$. Each terminal $j=1,2, \ldots, N$ has a superconducting phase $\phi_{j}$ and a supercurrent $I_{j}$. (b)-(e) SEM images of nanofabricated devices whose copies are used in this work. (b), (c) The $N=3$ case. (d) The $N=4$ case. (e) The multi-SQUID, where terminals 3 and 4 are short-circuited by a superconductor and the loop is pierced by a magnetic flux $\Phi$. (f) Junction's schematic cross section revealing the material stack and composition of each layer of the heterostructure (top) along with a TEM image of the semiconductor-superconductor interface (bottom).

critical current. In the $N=2$ case, the critical current is simply the maximal current that the junction can withstand while maintaining zero voltage. However, for $N=3$, there are two independent currents $I_{1}$ and $I_{2}$ externally supplied to the two terminals (the third one is grounded). The current combinations for which both terminals are at zero voltage define a simply connected two-dimensional region in the $\left(I_{1}, I_{2}\right)$ space. Thus, the scalar two-terminal critical current is replaced by a geometric object-the critical current contour (CCC) surrounding the zero-voltage region. For $N>3$, the zero-voltage region is surrounded by an $(N-1)$-dimensional hypersurface, whose visualization is impractical. However, for the here important case of $N=4$, the properties of the three-dimensional surface can be adequately reflected by its projections onto a set of twodimensional CCCs, obtained by imposing an external constraint on currents $I_{j}$ or phases $\phi_{j}$ of the terminals.

In this article, we report on the nontrivial geometric responses of the CCCs to the externally applied magnetic field and gate voltage, which is an inherent transport manifestation of multiterminal superconducting phase coherence. We implemented three species of hybrid semiconductor-superconductor devices: a three-terminal junction [Figs. 1(b) and 1(c)], a four-terminal junction [Fig. 1(d)], and a multiterminal superconducting quantum interference device, nicknamed "multi-SQUID" [Fig. 1(e)]. The multiSQUID is derived from a four-terminal junction by shortcircuiting terminals 3 and 4 with a superconducting wire, and it can be viewed as a flux-controlled three-terminal junction. Junctions with submicrometer dimensions are fabricated in a top-down fashion from an epitaxial heterostructure of the III-V group materials [49,50] [Fig. 1(f)]. By properly engineering the material stack, a high-mobility two-dimensional electron gas (2DEG) is confined near the crystalline interface between InAs semiconductor and a superconducting Al film. The superconducting terminals are electrically isolated from each other by mesa etching [Fig. 1(f)]. The junction's scattering region is defined by removing the Al layer. An important feature of our material is that the carrier density in the exposed semiconductor can be tuned all the way to a complete depletion using an electrostatic top gate (not shown in Fig. 1).

Our key experimental findings can be summarized as follows. In zero magnetic field, the CCC is necessarily smooth, inversion symmetric, and convex. Within such constraints, electrical top gating can deform the contour in a random fashion. A perpendicular magnetic field eliminates the contour's inversion symmetry and a sufficiently large field can introduce nodes: the curvature becomes discontinuous at seemingly random locations along the contour. A similar effect was observed in our multi-SQUID on tuning the flux through the loop toward the half-integer value, which is generated by orders of magnitude smaller field. However, the inversion symmetry recovers at the halfinteger flux bias $\Phi=h / 4 e$, where the CCC can acquire prominent shapes of, e.g., a six-pointed star.

The observed CCC geometries of our short junctions can be remarkably well reproduced using the scattering theory of Josephson effect generalized to the case of $N>2$. According to such a theory, the CCC is derived from the spectrum of the supercurrent-carrying ABS, whose energy is given by the Beenakker's determinant equation [51]:

$$
\operatorname{det}\left[1-\exp (-2 i \arccos (E / \Delta)) \hat{r} \hat{S}^{*} \hat{r}^{*} \hat{S}\right]=0 .
$$

Here $\hat{S}$ is the junction's normal scattering matrix, $E$ is the ABS energy, $\Delta$ is the superconducting gap in the terminals, 
and the matrix $\hat{r}=\exp \left(i \times \operatorname{diag}\left[\phi_{1}, \phi_{2}, \ldots, \phi_{N}\right]\right)$ incorporates the superconducting phases $\phi_{j}$ of all the terminals. Not knowing the junction's microscopic details, we used random ensembles of $\hat{S}$ matrices, constrained only by fundamental symmetries. Indeed, the zero-field CCC follows from the maximally constrained circular orthogonal ensemble (COE). Gating the semiconductor effectively samples the ensemble realizations. The effect of magnetic fields can be captured by switching to the circular unitary ensemble (CUE), which is appropriate in the absence of time-reversal symmetry. Finally, the multi-SQUID's CCC can be recreated at any flux $\Phi$ by incorporating the external constraint $\phi_{4}-\phi_{3}=\Phi \times 2 e / \hbar$ into the $\hat{r}$ matrix and using a single $\mathrm{COE} \hat{S}$ matrix.

Combining the results, our CCC collection provides a compelling evidence for the multiterminal Josephson effect. Our work thereby completes the first test of Eq. (1) beyond the case of $N=2$, on which many recent theory proposals are based. We expect that our measurement and analysis would guide the basic characterization of any other physical implementation of coherent multiterminal Josephson junctions.

The paper is organized as follows. In Sec. II, we show the raw transport data from three- and four-terminal junctions. Besides extracting the CCC, we establish that multiple Andreev reflection (MAR) is responsible for most of the differential resistance features outside the zero-voltage state. In Secs. III and IV, we describe the deformation of CCC in response to magnetic field and top gating, respectively. In Sec. V, we introduce the phase-sensitive multi-SQUID measurements. In Sec. VI, we model the data, and we conclude in Sec. VII.

\section{TRANSPORT IN THREE- AND FOUR- TERMINAL JOSEPHSON JUNCTIONS}

The three- and four-terminal junctions with submicrometer dimensions (Fig. 1) were fabricated using electron beam lithography and wet etching. In the first step, the aluminum film and quantum well layers are wet etched through a resist mask using Transene type $\mathrm{D}$ for $10 \mathrm{sec}$ and $\mathrm{H}_{2} \mathrm{O}: \mathrm{Ci}: \mathrm{H}_{3} \mathrm{PO}_{4}: \mathrm{H}_{2} \mathrm{O}_{2}(220: 55: 3: 3)$ for 4-8 min to define forklike and crosslike mesas, electrically isolated from each other by the few-hundred-nanometer deep cuts. In the second step, $\mathrm{Al}$ is removed from the desired junction region again using Transene type $\mathrm{D}$ at $50^{\circ} \mathrm{C}$. The fabrication procedure is similar to that used for two-terminal junctions [52]. The chips were wire bonded to a printed circuit board with built-in discrete-element filters, which was mounted to the copper probe of a bottom-loading dilution refrigerator. Experiments were performed at the base temperature between $10-20 \mathrm{mK}$. In the absence of a top gate, the exposed 2DEG in the junction region has the following approximate parameters, obtained through bulk transport measurements on a similar wafer [50]: Fermi wavelength
$\lambda_{F} \approx 25 \mathrm{~nm}$, velocity $v_{F} \approx 10^{6} \mathrm{~m} / \mathrm{s}$, mean free path $l_{e} \approx 200 \mathrm{~nm}$, and phase-breaking length $l_{\phi} \gtrsim 1 \mu \mathrm{m}$. For our devices with submicrometer dimensions, such parameters translate into the short-junction transport regime involving between 10 and 100 channels per terminal.

We start with the simpler case $N=3$ [devices similar to those shown in Figs. 1(b) and 1(c)]. Transport in threeterminal junctions is characterized by grounding the third terminal, and simultaneously measuring the voltage $V_{1}$ and $V_{2}$ of terminal 1 and 2 , as well as the differential resistance $d V_{1} / d I_{1}$ and $d V_{2} / d I_{2}$, as a function of the bias current $I_{1}$ and $I_{2}$. The data can be exhaustively summarized by three prominent features (Fig. 2). The junction's zero-voltage state in the $\left(I_{1}, I_{2}\right)$ plane can be graphically obtained as the intersection of the (blue colored) regions in Figs. 2(a) and 2(b), defined by the conditions $d V_{1} / d I_{1}=0\left(V_{1}=0\right)$ and $d V_{2} / d I_{2}=0\left(V_{2}=0\right)$, respectively. The CCC surrounds the zero-voltage region, as indicated in both Figs. 2(a) and 2(b) by a white dashed line. The second feature consists of the three radial directions along which the differential resistances of both terminals are reduced. This feature represents the special combinations of bias currents $I_{1}, I_{2}$, such that either $V_{1}=0$ or $V_{2}=0$ or $V_{1}=V_{2}$, and this is a generic property of dissipative transport in devices with multiple superconducting (a)

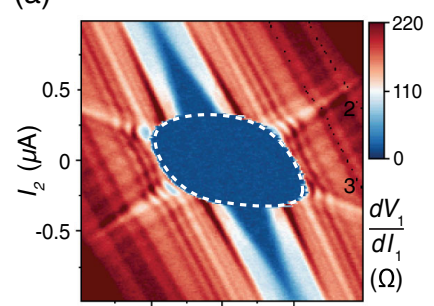

(b)

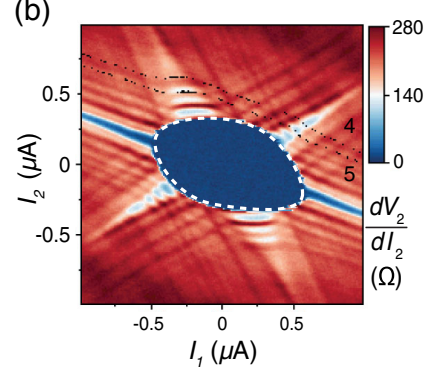

(c)

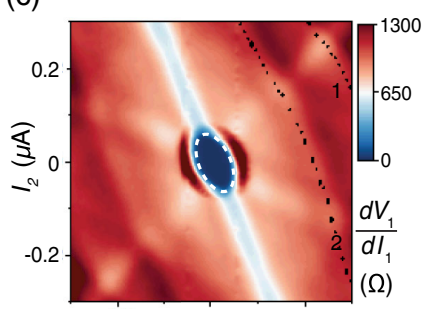

(d)

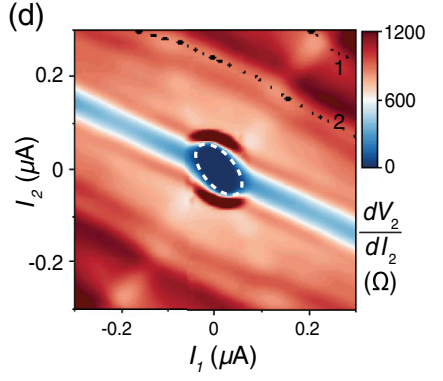

FIG. 2. Transport in three-terminal junctions. (a),(b) Differential resistance maps of the device shown in Fig. 1(b); terminal 3 is grounded. (c),(d) Same measurement for the device shown in Fig. 1(c). The CCC of both junctions are indicated by a dashed white line as a guide for the eye. The radial features in the resistance corresponding to the three conditions $V_{1}=0, V_{2}=0$, and $V_{1}-V_{2}=0$. Some of the MAR conditions $e V_{1}=2 \Delta / n$ (a),(c) and $e V_{2}=2 \Delta / n$ (b),(d) are shown as the black lines obtained using independently measured voltages $V_{1}$ and $V_{2}$ $(n=1,2, \ldots, 5)$. The third set of MAR corresponding to $e\left(V_{1}-V_{2}\right)=2 \Delta / n$ is not marked. The induced gap $\Delta$ is about $180 \mu \mathrm{eV}$ for both devices. 
terminals. The third feature is MAR resonances $[53,54]$ at positions in the $\left(I_{1}, I_{2}\right)$ plane given by $e\left|V_{1}\right|=2 \Delta / n$, $e\left|V_{2}\right|=2 \Delta / n$, and $e\left|V_{1}-V_{2}\right|=2 \Delta / n$, respectively, where $n$ is an integer and $\Delta \approx 180 \mu \mathrm{eV}$. Example equipotential lines corresponding to $n=2,3$ [Fig. 2(a)] and $n=4,5$ [Fig. 2(b)] are plotted on top of the $d V / d I$ data using independently measured voltage $V_{1}$ and $V_{2}$. Furthermore, we carefully verified the MAR conditions for $n=2,3, \ldots, 8$ by plotting the resistance data as a function of $2 \Delta / e V_{1}, 2 \Delta / e V_{2}$ (see Fig. $\mathrm{S} 1$ in the Supplemental Material [55]).

The presence of high-order MAR resonances along with the induced gap $\Delta$ being close to the gap of aluminum film indicates a nearly ballistic transport in the exposed semiconductor region and a high-transparency superconductorsemiconductor interface. Thus, the finite-voltage data confirm the high quality of both the heterostructure and the fabricated devices. Transport in the narrower leads junction [Fig. 1(c)] has all the same features described above [Figs. 2(c) and 2(d)]. The normal state conductance and the size of the zero-voltage region are reduced proportionally to the width of the leads. The MAR features are notably smeared, which may be the effect of scattering at the imperfect fabrication-defined edges. Nevertheless, a few lowest-order resonances are resolved and they give a similar value of the induced gap $\Delta \approx 180 \mu \mathrm{eV}$.

Having understood all the transport features of the threeterminal junctions, we move on to interpreting the more complex resistance maps of the four-terminal junctions (Fig. 3). Formally, the zero-voltage state of such devices [similar to the one shown in Fig. 1(d)] should be represented by a three-dimensional manifold in the space of the three independent bias currents (assuming one of the terminals is grounded). Instead, we pair the four terminals into three possible combinations $[(1,2),(3,4)],[(2,3),(4,1)]$, and $[(1,3),(2,4)]$ and apply symmetric currents to each pair, i.e., $I_{1}=-I_{3}=I_{13}$ and $I_{2}=-I_{4}=I_{24}$, etc. Consequently, the zero-voltage state can be characterized by three twodimensional CCCs defined in the planes of the currents $\left(I_{12}, I_{34}\right),\left(I_{23}, I_{41}\right)$, and $\left(I_{13}, I_{24}\right)$, respectively. The latter biasing configuration is particularly interesting, because the two currents $I_{13}$ and $I_{24}$ are forced to flow across each other in the central junction region. We use the differential resistance data taken in the $\left(I_{13}, I_{24}\right)$ plane in order to review the generic transport features encountered in our four-terminal junctions (Fig. 3).

Outside the zero-voltage region, there are six radial directions of reduced differential resistance, corresponding to zero voltage across one of the six terminal pairs [Figs. 3(a) and 3(b)]. Additionally, in the areas satisfying $\left|I_{13}\right| \gtrsim 1 \mu \mathrm{A}$ or $\left|I_{24}\right| \gtrsim 1 \mu \mathrm{A}$, there are prominent features in the differential resistance corresponding to the conditions $e\left|V_{12}\right|=2 \Delta, e\left|V_{23}\right|=2 \Delta$, etc. (not marked on the graph). Resistive transport at such high currents is likely influenced by the heating in the device and it is of little
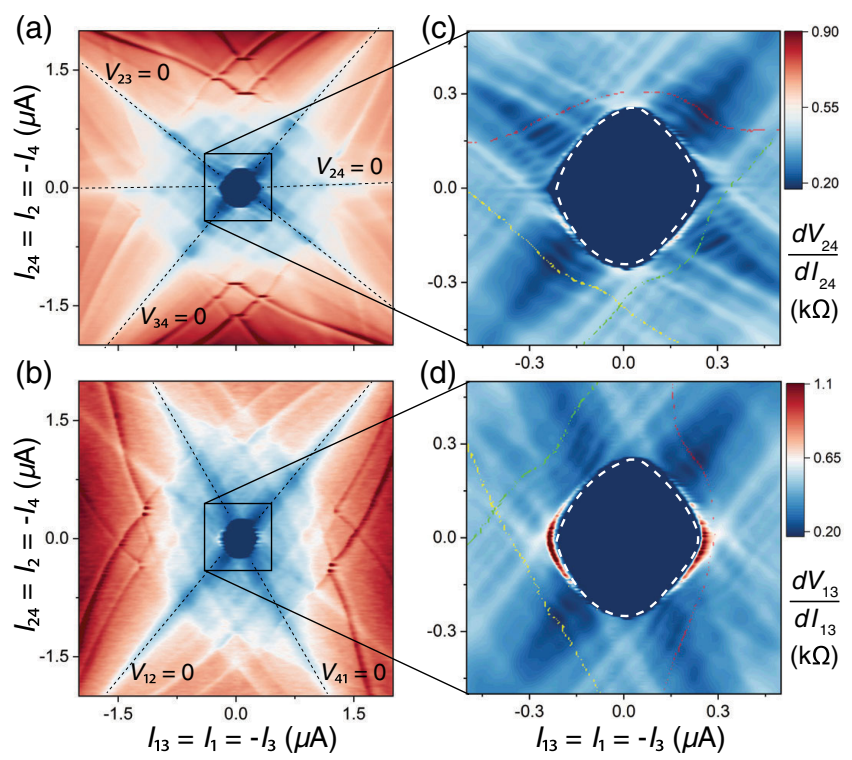

FIG. 3. Transport in a four-terminal junction. (a),(b) Differential resistance across the terminal pair $(2,4)$ and $(1,3)$ in the device shown in Fig. 1(d). (c),(d) Enlargement of the zero-voltage region. The CCCs are marked by a white dashed line as a guide to the eye. The six radial features represent the conditions $V_{12}=0$, $V_{23}=0$, etc., as marked directly on the plots. The colored lines represent some of the MAR conditions. (c) Red, $e V_{24}=2 \Delta / n$; yellow, $e V_{23}=2 \Delta / n$; green, $e V_{34}=2 \Delta / n$. (d) Red, $e V_{13}=$ $2 \Delta / n$; yellow, $e V_{41}=2 \Delta / n$; green, $e V_{12}=2 \Delta / n$. Here $\Delta \approx$ $160 \mu \mathrm{eV}$ and $n=5$.

interest here. Similarly to the three-terminal junctions, the zero-voltage region in the $\left(I_{13}, I_{24}\right)$ plane satisfies $d V_{13} / d I_{13}=d V_{24} / d I_{24}=0$. We have checked that such a condition is indeed sufficient to ensure that the voltage between all the six terminal pairs is zero (see Fig. S2 in the Supplemental Material [55]). The resulting CCC is emphasized by a white dashed line [Figs. 3(c) and 3(d)].

Immediately outside the $\mathrm{CCC}$, there is an intricate structure in the differential resistance [Figs. 3(c) and 3(d)]. We believe most of this structure can also be explained by MAR involving the voltage drops across the six possible terminal pairs. For example, using independently measured voltages, we indicate the equipotential lines in the $\left(I_{13}, I_{24}\right)$ plane corresponding to the $(n=5)$ MAR conditions directly on top of the data in Figs. 3(c) and $3(d)$. Equipotentials corresponding to $(n=2-8)$ are shown in Fig. S3 [55]. Notably, we found evidence for the eighthorder MAR involving the more separated terminal pairs $(1,3)$ and $(2,4)$. Such an observation confirms that every pair of terminals in our four-terminal junction is connected by transparent channels across the semiconductor.

\section{EFFECT OF MAGNETIC FIELD ON CCC}

In Fig. 4(a) we show the effect of a perpendicular magnetic field $B$ on transport in the same four-terminal junction described in Sec. II. The magnetic field was 
applied using an externally mounted handmade superconducting coil. The differential resistance across the terminal pairs $(1,3),(1,2)$, and $(4,1)$ was measured at $B=0$ and up to $B=2 \mathrm{mT}$ as a function of the three possible symmetric current pairs $\left(I_{13}, I_{24}\right),\left(I_{12}, I_{34}\right)$, and $\left(I_{41}, I_{23}\right)$, respectively. Resistance of the complementary pairs $(2,4),(3,4)$, and $(2,3)$ was also measured (see Fig. S4 in the Supplemental Material [55]). The field $B=2 \mathrm{mT}$ dramatically modifies the zero-voltage region in each of the three maps [Fig. 4(a), dark blue]. Such a field corresponds to approximately $1 / 5$ of the flux quantum piercing the exposed semiconductor in the junction, although in reality the flux is probably larger due to the flux focusing effect. We avoided measuring at a higher field as it starts to gradually suppress superconductivity in aluminum.

The CCCs at different $B$ values were extracted using the method described in Sec. II. In Fig. 4(b) we visualize each of the three extracted CCCs by painting in colors the zerovoltage region. The first column of Fig. 4(b) compares the CCC at $B=0$ (light blue) and $B=1.2 \mathrm{mT}$ (solid blue). At zero field, all the three contours are convex and symmetric with respect to inversion around the origin. The field $B=1.2 \mathrm{mT}$ distorts the CCCs such that they are still convex but no longer inversion symmetric. A qualitatively new feature arises on increasing the field to $B \approx 1.6 \mathrm{mT}$ : the local curvature changes its sign around several points of the contour. For even stronger field $B=2 \mathrm{mT}$ such features become more pronounced. As a sanity check, we show that flipping the sign of magnetic field is equivalent to inverting the contours around the origin [Fig. 4(b), last column]. Note that although the contour's geometry in the presence of the $B$ field is qualitatively different, their total area reduced only by about a factor of 2. Such dramatic sensitivity of the CCC geometry to magnetic flux piercing the semiconductor is indicative of the phase-coherent supercurrent flow across the multiple terminals of the junction.

\section{EFFECT OF TOP GATING ON CCC}

One advantage of our specific hybrid semiconductorsuperconductor materials platform is that the carrier density in the junction's scattering region can be tuned using a top gate. This was achieved by depositing a thin dielectric layer on top of the prefabricated four-terminal junction device similar to that shown in Fig. 1(d) and then covering the junction completely by a metal film electrode which is the top gate. The gate electrode consists of a 5-nm Ti adhesion (a)
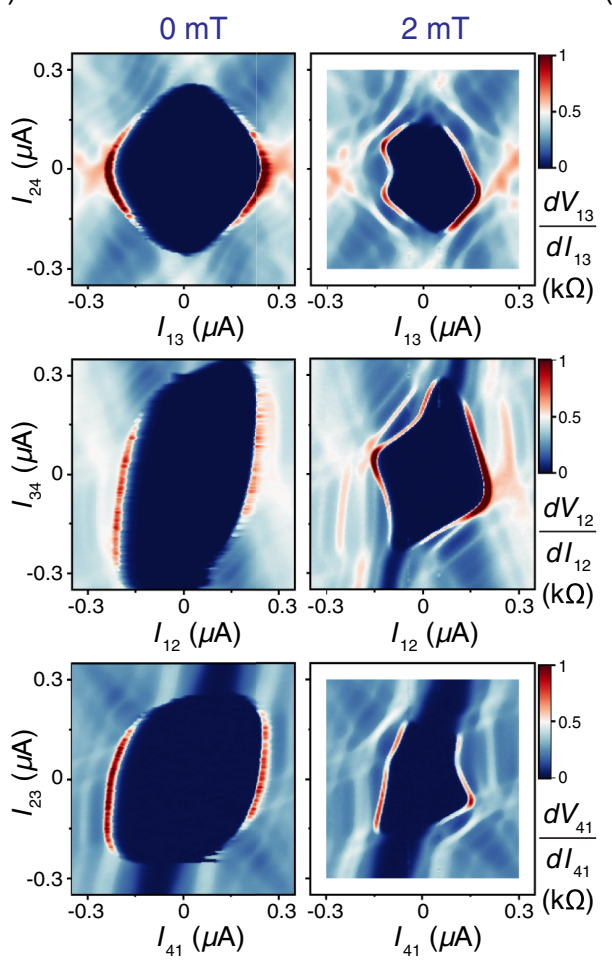

(b)
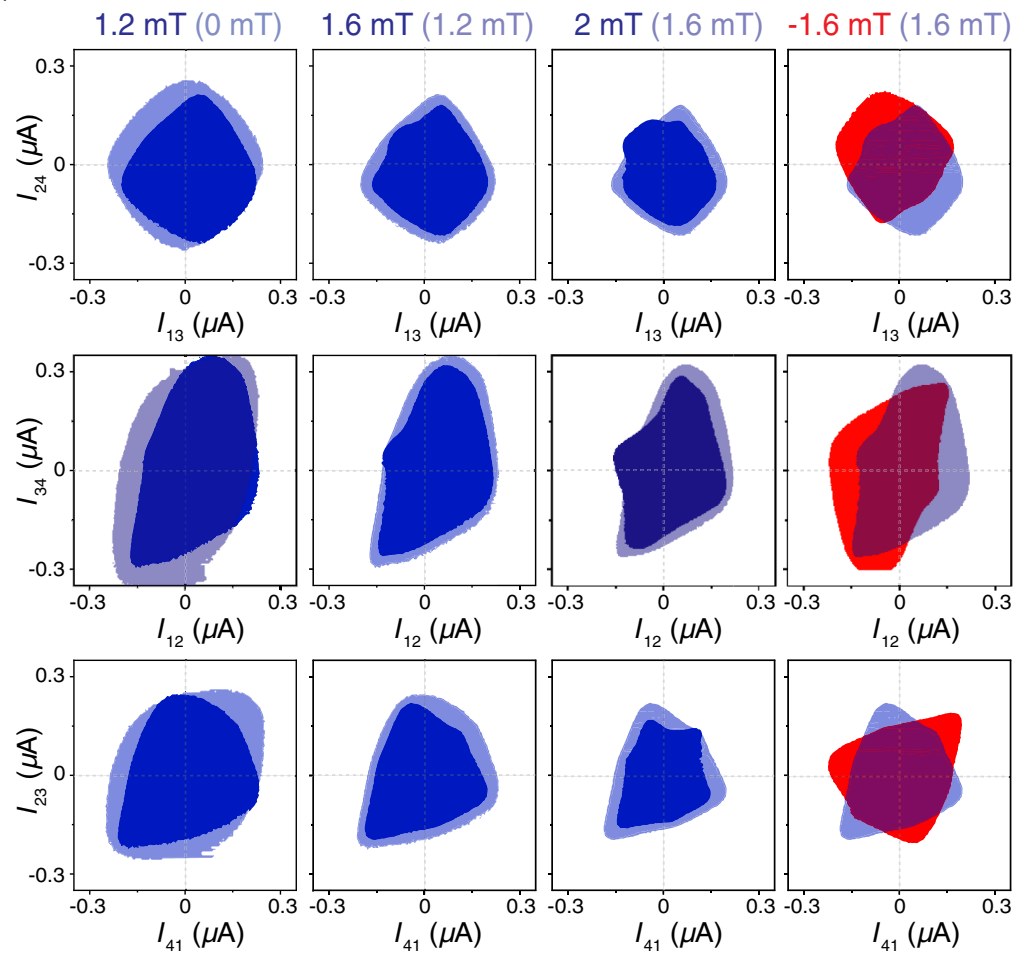

FIG. 4. Effect of magnetic field on a four-terminal junction. (a) Differential resistance maps of a symmetrically biased four-terminal junction measured at $B=0$ (left) and $B=2 \mathrm{mT}$ (right). The three rows represent the three symmetric bias current-current planes $\left(I_{13}, I_{24}\right),\left(I_{12}, I_{34}\right)$, and $\left(I_{41}, I_{23}\right)$. The maps of the resistance of the complementary terminal pairs $(2,4),(3,4)$, and $(2,3)$ are not shown. (b) Evolution of CCC in a magnetic field. The extracted zero-voltage regions, which the CCCs surround, are depicted by a color. Note the comparison of data at $B=1.6 \mathrm{mT}$ (transparent blue) versus $B=-1.6 \mathrm{mT}$ (transparent red), which is equivalent to inversion of the $\mathrm{CCC}$ around the origin. 
layer and a 100-nm Au electrode layer. A 50-nm-thick aluminium oxide film was deposited using atomic layer deposition to couple the semiconductor to the gate electrode. As a result, the application of a sufficiently large negative voltage (around $-5 \mathrm{~V}$ for this device) to the top gate completely suppresses the conductance across every terminal's pair.

In Fig. 5, we show the evolution of CCC in a top-gated four-terminal junction at three different gate voltages $V_{g}$. We focus on the differential resistance data in the $\left(I_{13}, I_{24}\right)$ plane as we did for the nongated device in Fig. 3. In the absence of a magnetic field, at $V_{g}=-4.3 \mathrm{~V}$, the CCC has a similar shape to that of the device shown in Fig. 3: it obeys the inversion symmetry but it is notably asymmetric with respect to flipping one of the two axis. Moving to $V_{g}=-4.5 \mathrm{~V}$ deforms the CCC into nearly an oval stretched along the $I_{13}$ axis. Moving a bit further down in gate voltage to $V_{g}=-4.55 \mathrm{~V}$, the CCC becomes nearly a circle with a radius of about $30 \mathrm{nA}$. Such a current scale corresponds to the critical current of a single transparent channel. On the other hand, the product of such a current scale with the normal resistance scale (a few kilohm) is about $100 \mu \mathrm{V}$, and this is close to the value of the induced gap $\Delta \approx 180 \mu \mathrm{V}$. Thus, we conclude that top gating can readily bring our junctions into the regime of one or a few scattering channels per terminal.

Interestingly, there appears to be no obvious correspondence between the three applied gate voltages and the resulting geometries of the CCC. Besides the two constraints of inversion symmetry and convexity, the CCC geometry changes more or less at random with the gate voltage. Application of magnetic fields also deforms the three contours in a seemingly random fashion. For the largest-area CCC at $V_{g}=-4.3 \mathrm{~V}$ and $B=2 \mathrm{mT}$, the field does induce the nonconvex feature similar to that reported in Fig. 4. The field-induced deformations of the CCC break the inversion symmetry, and the resulting shapes at $V_{g}=-4.5 \mathrm{~V}$ and $V_{g}=-4.55 \mathrm{~V}$ appear completely unrelated to their zero-field versions. A detailed study of gating of multiterminal junctions will be reported elsewhere. For the purpose of this work, we conclude that the application of a gate voltage is equivalent to creating a new device or switching the device off when the magnitude of negative bias exceeds the threshold.

\section{EFFECT OF PHASE BIAS ON CCC}

The ultimate manifestation of superconducting phase coherence in the conventional Josephson effect is the flux modulation of the supercurrent in a SQUID. A conventional SQUID consists of two Josephson junctions in a superconducting loop pierced by magnetic flux. The supercurrents flowing in each arm can interfere constructively or destructively depending on the magnetic flux. The supercurrent interference in the multi-SQUIDs is more complex [Fig. 1(e)]. Here the external magnetic flux $\Phi$ piercing the loop establishes the phase difference $\phi_{4}-\phi_{3}=\Phi \times 2 e / \hbar$ while the current is applied to the other two terminals 1 and 2 . In case the loop is floating, the current $I_{1}$ flowing into terminal 1 equals the current $-I_{2}$ flowing into terminal 2 (a) $V_{g}=-4.3 \mathrm{~V}$
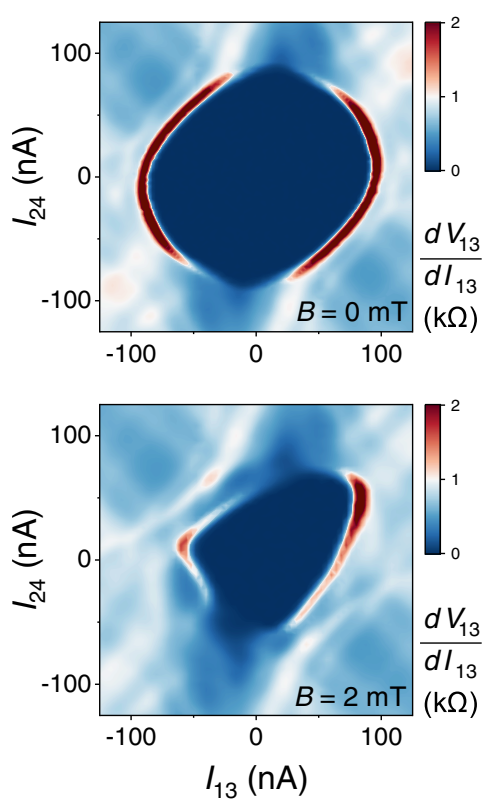

(b) $V_{g}=-4.5 \mathrm{~V}$
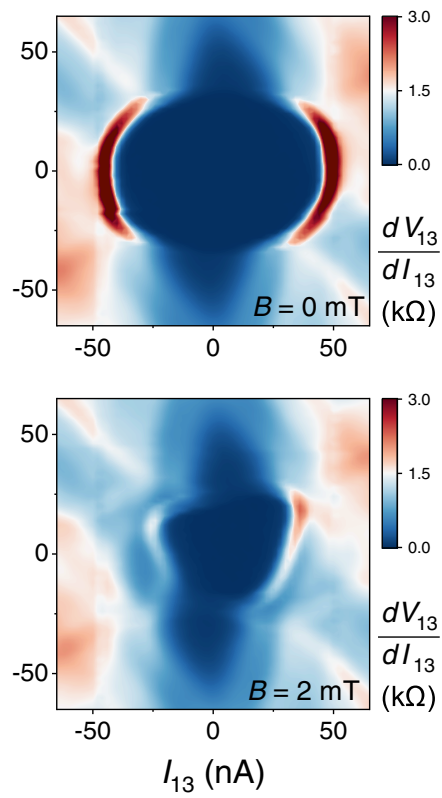

(c) $V_{g}=-4.55 \mathrm{~V}$
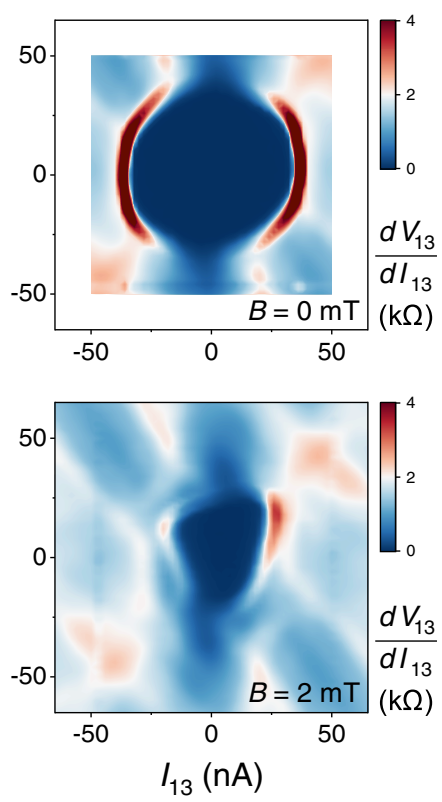

FIG. 5. Effect of top gating on a four-terminal junction. (a)-(c) Differential resistance map in the $\left(I_{13}, I_{24}\right)$ plane taken at three values of the top-gate voltage at $B=0$ (top row) and at $B=2 \mathrm{mT}$ (bottom row). 
due to the current conservation, so we can define $I_{12}=I_{1}=-I_{2}$. In such a biasing configuration the multiSQUID resembles a conventional SQUID in the sense that it is a flux-controlled two-terminal superconducting device. However, in case the loop is grounded, the current $I_{1}$ and $I_{2}$ can be set independently, and the multi-SQUID becomes a flux-controlled three-terminal junction.

In the first test, we float the loop and measure the differential resistance $d V_{12} / d I_{12}$ as a function of the bias current $I_{12}$ and the flux $\Phi$ through the loop [Fig. 6(a)]. During the measurement, we swept up the positive current and swept down the negative current from zero in order to avoid a small retrapping effect in the switching dynamics. Flux-periodic oscillations of $d V_{12} / d I_{12}$ are clearly visible while the critical current is notably asymmetric with respect to positive and negative values, with the exception of the integer and the half-integer flux. It is still symmetric under the current and flux inversion $(I \rightarrow-I, \Phi \rightarrow-\Phi)$. Our observation can be understood as a result of the superconducting phase-drag effect [56]: the externally set phase difference $\phi_{4}-\phi_{3}$ induces a phase difference between terminals 1 and 2, which periodically offsets the maximally allowed value of supercurrent $I_{12}$. Moreover, in case of terminals 1 and 2 also being connected by another superconducting loop, the phase-drag effect would result in the magnetic flux transfer between the loops [5,6,57].

In a more elaborate multi-SQUID experiment, we ground the loop (terminals 3 and 4), and treat the device as a three-terminal junction. Repeating the procedures described in Sec. II, we measure the resistance maps of $d V_{1} / d I_{1}$ and $d V_{2} / d I_{2}$ as a function of $\left(I_{1}, I_{2}\right)$ and $\Phi$. While the familiar shape of the CCC at a zero flux is similar to that of a regular three-terminal junction, the half-integer flux introduces a dramatic modification: the CCC acquires the shape of a skewed six-pointed star, which preserves inversion symmetry. Note that the flux bias expectedly does not affect the transport at nonzero voltage. The clearly visible MAR resonances have a similar pattern to those shown in Fig. 2, and they merely shift in the $\left(I_{1}, I_{2}\right)$ plane in order to adjust to the modified geometry of the CCC (see Fig. S5 in the Supplemental Material [55]). We have tracked the evolution of CCC as a function of flux [Fig. 6(c)] which results in a similar deformation to that induced by the magnetic field described in Sec. III and Fig. 4. Yet, the magnetic field scale in the multi-SQUID experiment is orders of magnitude lower. We conclude that piercing magnetic flux through the scattering region and flux biasing the loop of a multi-SQUID leads to similar in nature multiterminal superconducting interference effects.

\section{EXPERIMENT VERSUS THEORY}

For a given scattering matrix $\hat{S}$ of a short junction, the $\mathrm{CCC}$ can be obtained numerically from Eq. (1) using the following procedure. First, the junction's ground state energy $E_{g}$ is calculated from the ABS energies according (a)

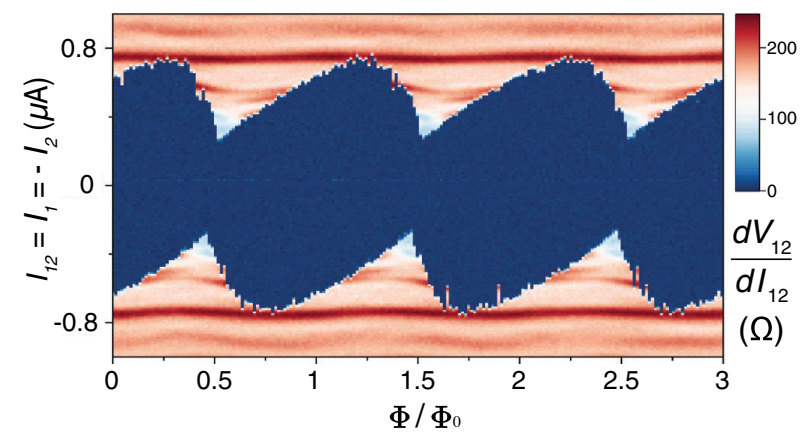

(b)

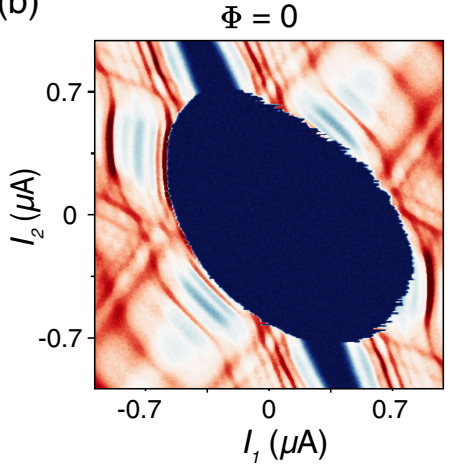

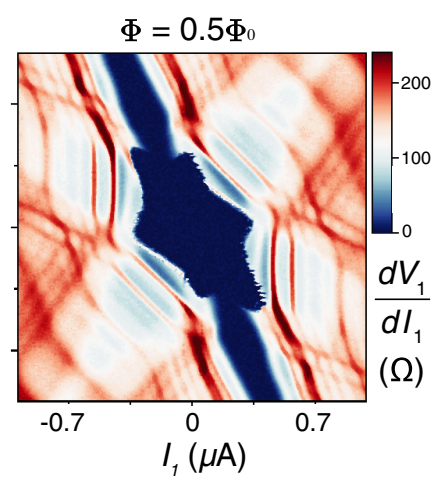

(c)
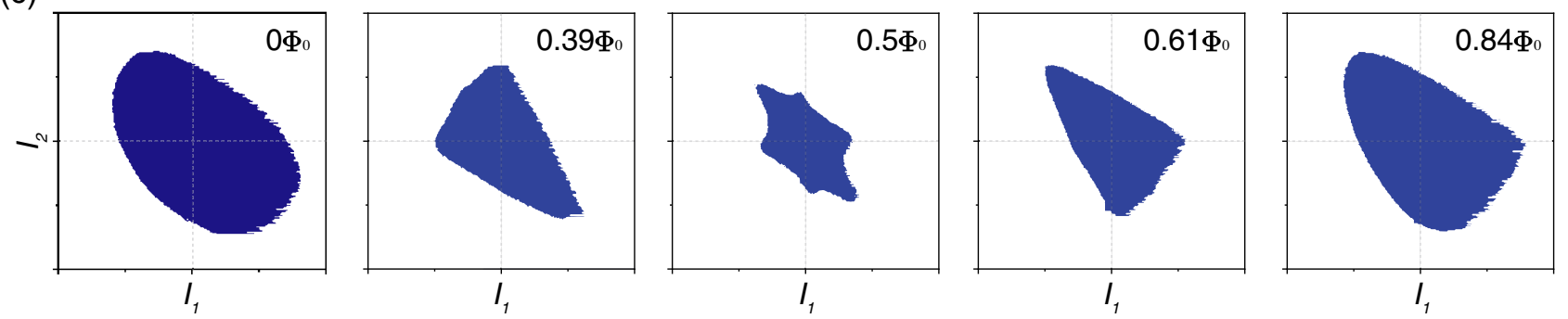

FIG. 6. Phase-drag effect and phase-controlled CCC in a multi-SQUID. (a) Differential resistance between terminal 1 and 2 as a function of the flux $\Phi$ through the loop in the device shown in Fig. 1(e); the loop is floating. $\Phi_{0}=h /(2 e)$ is the superconducting flux quantum. (b) Differential resistance of the terminal 1 as a function of the two currents $I_{1}, I_{2}$, for an integer flux (left) and a half-integer flux (right) though the loop; the loop is grounded. (c) Evolution of the CCC as a function of flux through the loop. Note the formation of an unusual inversion-symmetric star shape at the half-integer flux bias. 
to $E_{g}=-1 / 2 \sum_{n} E_{n}$, where $E_{n}$ is the $n$th positive solution of Eq. (1). In the general case of an $N$-terminal junction, $E_{g}$ is a function of $N-1$ phase differences. When the junction is driven with bias currents $I_{1}, \ldots, I_{N-1}$, the free energy $F$ is given by

$F\left(\phi_{1}, \ldots, \phi_{N-1}\right)=E_{g}\left(\phi_{1}, \ldots, \phi_{N-1}\right)-\frac{\hbar}{2 e} \sum_{i=1}^{N-1} I_{i} \phi_{i}$

For the junction to be in the zero-voltage state, there should exist a stable equilibrium position of the free energy. It requires both $\nabla F=0$ and positiveness of the Hessian eigenvalues. Then, the CCC is obtained from a boundary of allowed bias currents $I_{1}, \ldots, I_{N-1}$.

This is the $(N-1)$-dimensional tilted washboard potential problem, which is used in general to describe the current-biased Josephson junctions. Unlike calculating the critical current of two-terminal Josephson junctions, the second derivatives of the free energy need to be investigated to construct the $\mathrm{CCC}$ in the multiterminal Josephson junctions.

Within the framework of Eq. (1), the microscopic junction details are conveniently hidden inside the scattering matrix $\hat{S}$. Such an approach is relevant for our "short" junctions, where the scattering phases change slowly with energy. In the simplest case of a two-terminal junction with one or two transport channels, the corresponding transmission coefficients can be used as adjustable parameters to fit the experimental data. However, having four terminals with each containing at least a few channels, the size of the scattering matrix becomes too large to consider its elements individually. In the spirit of mesoscopic physics,

(a) $\mathrm{COE}$

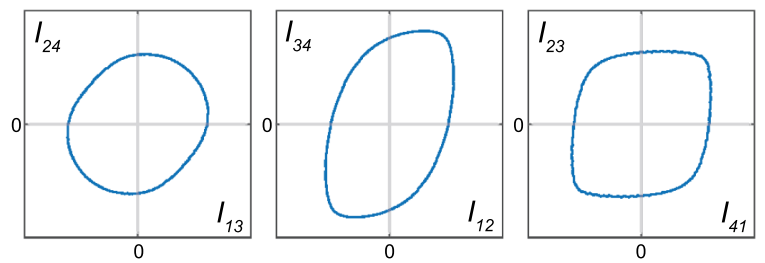

(c)
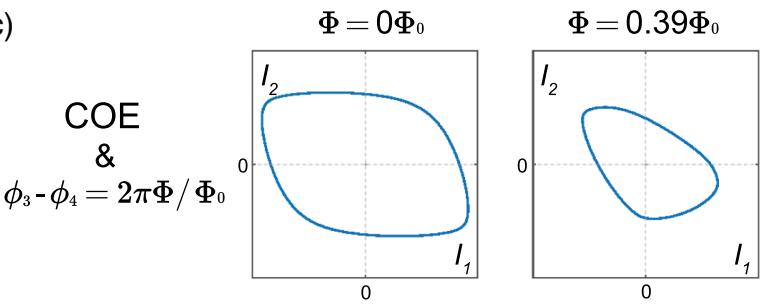

we consider random $\hat{S}$ matrices, restricting their structure only by fundamental symmetries. There are two choices of random matrix ensembles appropriate to describe our system: the circular orthogonal ensemble, relevant in the presence of both time-reversal and spin-rotation symmetries, and the circular unitary ensemble, relevant in the absence of time-reversal symmetry. The third circular symplectic ensemble (CSE) has time-reversal symmetry but lacks spin-rotational symmetry. It turns out that within the framework of Eq. (1), the CSE and COE ensembles are indistinguishable in their effect on the CCC geometry.

The simulated CCCs using a random scattering matrix are presented in Fig. 7. The zero-field data of Fig. 4, i.e., all the three CCC of a symmetrically biased four-terminal junction, can be readily recreated using a single COE scattering matrix. Likewise, it was also straightforward to find COE $\hat{S}$ matrices reproducing the contour deformations induced by the top-gate voltage in Fig. 5. In fact, absence of nodes and inversion symmetry of the contours is directly related to the orthogonality constraint on the matrix elements, and the CCC remains smooth even in the case of a single scattering channel. Eliminating the orthogonality constraint in the CUE matrices immediately introduces the kind of nonconvex contours with nodes [Fig. 7(b)] that we observed in the presence of a sufficiently large perpendicular magnetic field (as shown in Fig. 4). Moreover, the relative reduction of the area enclosed by the CCC upon switching from COE to CUE approximately matches the data as well. Even more confidence in our model comes from matching the CCC of a multi-SQUID at various flux-bias values using a single COE matrix [Figs. 7(c) and 6(c)]. The magnetic field in the junction region is essentially zero in this case, but the flux $\Phi$ through

(b) CUE
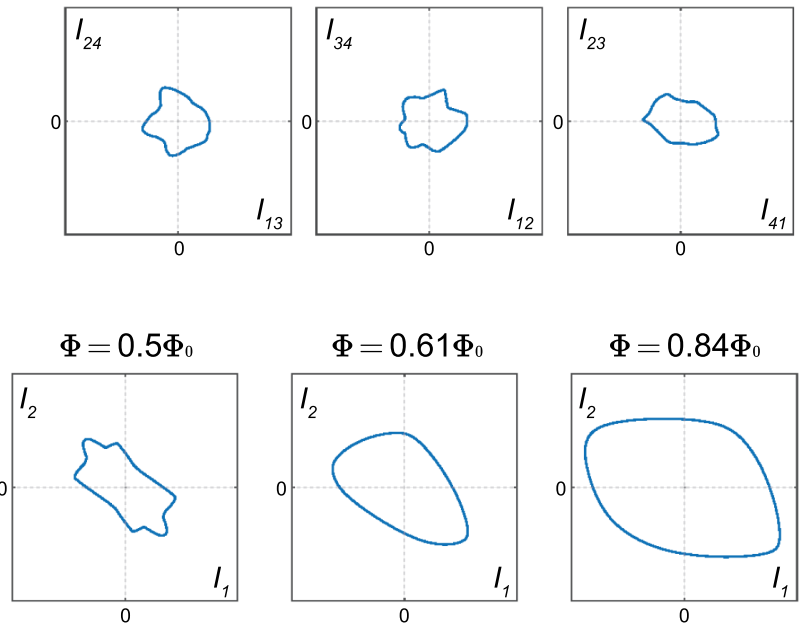

FIG. 7. Numerical modeling of CCC. (a) The CCC of a symmetrically biased four-terminal junction using a single COE $\hat{S}$ matrix. Note the similarity with the measured contours in Fig. 4. (b) Same as (a) produced using a CUE matrix. In both (a) and (b) plots the scattering matrices have the same size and the current scale is kept the same. (c) Evolution of the CCC of a multi-SQUID as a function of flux $\Phi$ through the loop produced using a single $\operatorname{COE} \hat{S}$ matrix. 
the loop sets a phase difference $\phi_{3}-\phi_{4}=\Phi \times 2 e / \hbar$. Such a constraint can be directly incorporated into the $\hat{r}$ matrix of Eq. (1), which makes the ABS spectrum and hence the CCC flux-dependent.

The emergence of nodes along the $\mathrm{CCC}$ in the presence of magnetic field [Fig. 7(b)] and phase bias [Fig. 7(c)] can be more deeply understood by mapping the contours back to the space of the phase differences (Fig. 8). For simplicity, we restrict the discussion to three-terminal junctions, assuming terminal 3 is grounded. The CCC in the $\left(I_{1}, I_{2}\right)$ plane is equivalent to the steepest gradients of $E_{g}(\boldsymbol{\phi})$ in each gradient direction of $\boldsymbol{\phi}=\left(\phi_{1}, \phi_{2}\right)$ in the region of the two Hessian eigenvalues being positive. Since $E_{g}(\boldsymbol{\phi})$ is a periodic continuous function, there is at least one local minimum within the Brillouin zone $[0,2 \pi] \times[0,2 \pi]$. In the absence of a magnetic field, $E_{g}$ always has a local minimum at zero phases, $\phi_{1,2}=0$, surrounded by the points that give steepest gradients in each gradient direction and two positive Hessian eigenvalues. In this case, the CCC is inversion symmetric and convex [see Fig. 8(a)].

When the magnetic flux penetrates the junction, an additional phase adds up in the wave function of electrons and holes propagating through the junction. As a result, $\phi_{1,2}=0$ is not a special point and $E_{g}$ can have multiple local minima; see Fig. 8(b). In this case, the steepest gradients around each minima in the region of positive Hessian eigenvalues would still form a single close loop, but the critical current is determined by the maximal values among these loops for a specific direction of the current in the $\left(I_{1}, I_{2}\right)$ plane. Such maximal values may switch from one loop around minima to another loop. This switching between different loops in the space of phase differences [Fig. 8(b), top] gives rise to nodes in the corresponding CCC [Fig. 8(b), bottom]. Because of smoothing of both experimental and numerical data, the nodes appear as sharper features of the contour with local curvature changing its sign.

In the case of a multi-SQUID, $E_{g}$ is also a function of only two phases, $\phi=\left(\phi_{1}, \phi_{2}\right)$. The externally set phase $\phi_{3}$ (we set $\phi_{4}=0$ ) plays qualitatively the same role as switching the matrix ensemble from COE to CUE. At $\phi_{3}=0$ (integer flux bias), the landscape of $E_{g}$ is similar to that of a three-terminal junction, with a single minimum at $\phi_{1,2}=0$, which gives a convex CCC [Fig. 8(c)]. However, $\phi_{3} \neq 0$ deforms the ground state energy landscape, producing additional minima [Fig. 8(d)]. Consequently, the CCC breaks into separate disconnected pieces in the phase space and develops cusps in the current space. The half-flux quantum bias is special because then $\phi_{3}=\pi$ and the $\operatorname{exponent} \exp \left(i \phi_{3}\right)=-1$ in Eq. (1) is real. In this case, $E_{g}$ acquires two symmetric minima in the Brillouin zone [Fig. 8(e)] which break up the $\mathrm{CCC}$ in the phase space in a symmetric manner, leading to the prominent time-reversal-symmetric CCC in the shape of a six-pointed star. (a) $3 \mathrm{~T}-\mathrm{COE}$
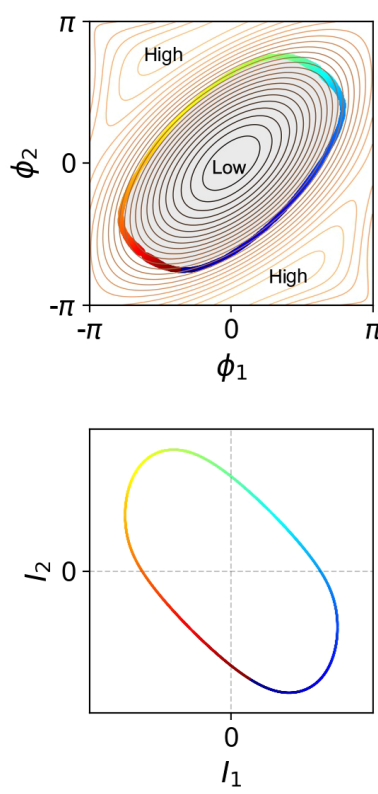

(b) 3T-CUE
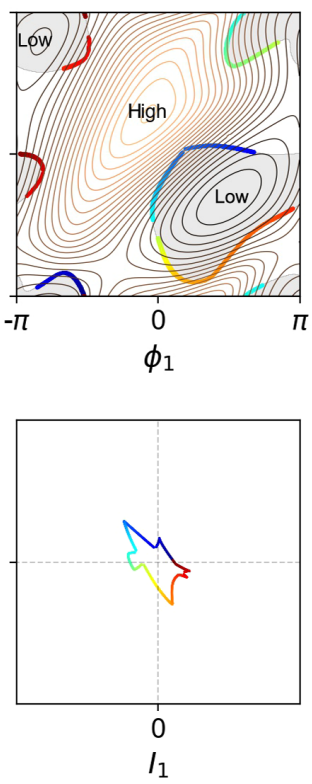

(c) $4 \mathrm{~T}-\mathrm{COE}$ $(\Phi=0)$
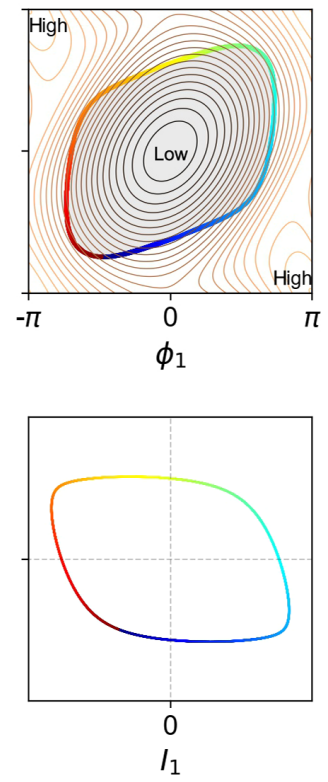

(d) $4 \mathrm{~T}-\mathrm{COE}$ $\left(\Phi=0.45 \Phi_{0}\right)$
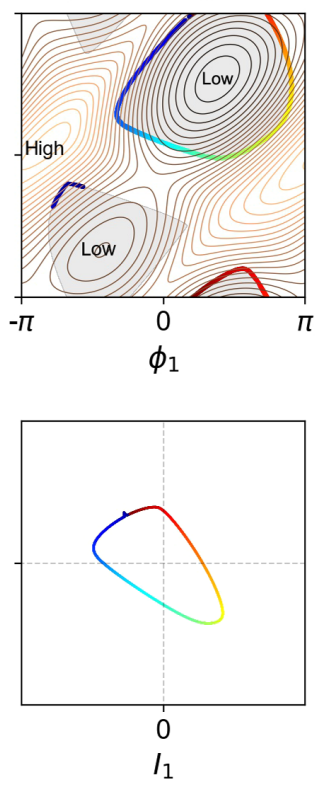

(e) $4 \mathrm{~T}-\mathrm{COE}$ $\left(\Phi=0.5 \Phi_{0}\right)$
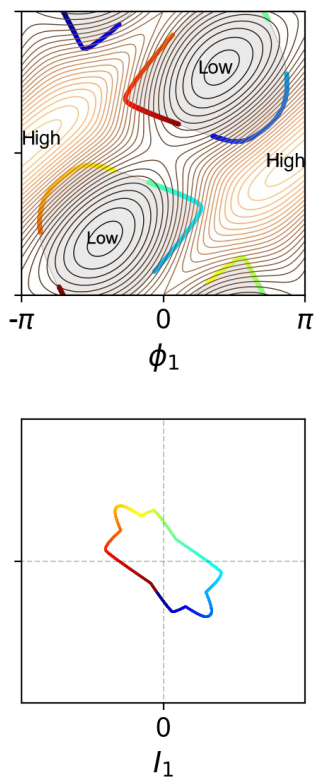

FIG. 8. Partitioning of CCC in the phase space. (Top) Contour map of the ground state energy and (bottom) the corresponding CCC of the three-terminal junctions without a loop and four-terminal junctions with a loop. (a) Three-terminal (3T), no $B$ field, (b) Threeterminal (3T), nonzero $B$ field, (c) Four-terminal (4T) with a loop $\Phi=0$, (d) $\Phi=0.45 \Phi_{0}$, (e) $\Phi=0.5 \Phi_{0}$. The CCC is a boundary of all possible configurations of $\left(I_{1}, I_{2}\right)$. Each configuration $\left(I_{1}, I_{2}\right)$ of color on the CCC is given by the phase values $\left(\phi_{1}, \phi_{2}\right)$ of the same color in the corresponding energy contour map. The shaded area is where the two Hessian eigenvalues are positive. 
Finally, let us stress that one does not expect coherent multiterminal scattering to occur in junctions with too wide leads. In that case, the scattering matrix in Eq. (1) loses its multiterminal structure and breaks into blocks representing two-terminal scattering. As a consequence, the $\mathrm{CCC}$ would lose the geometric properties summarized in Fig. 7. We have fabricated intentionally wide four-terminal junctions (width of $3 \mu \mathrm{m}$ ) and we used the gate voltage to reduce the normal conductance scale to that of our small coherent junction devices. Indeed, the CCCs of such wide junctions are dramatically different from those reported here (Fig. S6 [55]), but they bear similarities to those reported in two recent experiments [44,58]. The contours are no longer convex in zero field and they shrink on increasing the field while keeping their inversion symmetry. In this case, supercurrent transport can be modeled using a network of two-terminal Josephson junctions, which is equivalent to the Beenakker's scattering matrix approach with block-diagonal scattering matrices coupling different pairs of terminals. Thus, we observe a good agreement between theoretical and experimental CCC for a very diverse set of device geometries.

\section{SUMMARY AND OUTLOOK}

We introduced a new concept of the critical current contour - the key ground state characteristic of a multiterminal Josephson junction. Such a geometric object is readily available from an elementary transport measurement, yet it remained overlooked in previous theoretical and experimental studies. To test the new concept, we created a variety of novel junction devices, including the very first demonstration of the multi-SQUID. The CCC undergoes nontrivial deformations in response to electrostatic gating, magnetic field, and phase bias, which are the three external stimuli usually used to probe the Josephson effect in two-terminal junctions. Yet, all our observations are reproduced remarkably well by the scattering theory of multiterminal Andreev bound states, which makes no assumptions about the junction's microscopic details. It is thus probable that our data represent a generic manifestation of the Josephson effect in mesoscopic junctions of more than two superconductors.

Our experiment was made possible by the favorable properties of the epitaxial InAs/Al heterostructure: transparent semiconductor-superconductor interface, high mobility of the exposed surface 2DEG, and an overall robustness with respect to the device fabrication procedures. Even more intriguing developments are expected with such a materials platform in the context of recently proposed experiments on multiterminal Josephson junctions. As already noted here, gating the carrier density in the semiconductor allows depleting the transport into the single channel regime. In this case the junction's ABS energies as a function of multiple phase differences can be individually resolved in a tunneling experiment [59]. Such a measurement would test, e.g., predictions of the Weyl nodes physics in four-terminal junctions [9]. Moreover, our material was also shown to have a strong spin-orbit coupling, resulting in a recently reported evidence of the induced $p$-wave order in the presence of an in-plane magnetic field [60]. With our work all components are now in place to explore trijunctions of topological superconductors, required for braiding of Majorana fermions [22,23,61]. Finally, gated three- and four-terminal junctions can act as superconducting digital devices [62], tunable superconducting transformers, and nonlinear elements, which can be useful for controlling superconducting quantum bits [63] and creating quantumlimited sensors and amplifiers [64]. In particular, replacing certain multijunction elements, such as Josephson ring modulators [65] and Josephson rhombus chains [66] by multiterminal Josephson junctions introduces a potentially useful resource to the quantum circuits toolbox.

\section{ACKNOWLEDGMENTS}

We thank Alex Levchenko and Anton Akhmerov for helpful discussions about critical current contours. We acknowledge funding from National Science Foundation (NSF) Physical Frontier Center (PFC) at the Joint Quantum Institute (No. 1430094), NSF Division of Materials Research (DMR) (No. 1836687), Defence Advanced Research Projects Agency (DARPA) Young Faculty Grant (No. D17AP00025), and United States Army Research Office (US ARO) program "New and Emerging Qubit Science and Technology" (No. W911NF1810115).

[1] B. D. Josephson, Possible New Effects in Superconductive Tunnelling, Phys. Lett. 1, 251 (1962).

[2] I. O. Kulik and A. N. Omel'yanchuk, Josephson Effect in Superconductive Bridges: Microscopic Theory, Sov. J. Low Temp. Phys. 4 296, (1978).

[3] Yu. G. Bevza, V. I. Karamushko, E. A. Kel'man, G. G. Tsach, and I. M. Dmitrenko, Electrically Controlled Josephson Elements, Sov. J. Low Temp. Phys. 5 (1979).

[4] R. de Bruyn Ouboter, A. N. Omelyanchouk, and E. D. Vol, Multi-Terminal Squid Controlled by the Transport Current, Physica (Amsterdam) 205B, 153 (1995).

[5] M. Amin, A. N. Omelyanchouk, and A. M. Zagoskin, dc Squid Based on the Mesoscopic Multiterminal Josephson Junction, Physica (Amsterdam) 372B, 178 (2002).

[6] M. Amin, A. N. Omelyanchouk, A. Blais, A. M. van den Brink, G. Rose, T. Duty, and A. M. Zagoskin, Multi-Terminal Superconducting Phase Qubit, Physica (Amsterdam) 368C, 310 (2002).

[7] B. van Heck, S. Mi, and A. R. Akhmerov, Single Fermion Manipulation via Superconducting Phase Differences in Multiterminal Josephson Junctions, Phys. Rev. B 90, 155450 (2014).

[8] T. Yokoyama and Y. V. Nazarov, Singularities in the Andreev Spectrum of a Multiterminal Josephson Junction, Phys. Rev. B 92, 155437 (2015). 
[9] R.-P. Riwar, M. Houzet, J. S. Meyer, and Y. V. Nazarov, Multi-Terminal josephson Junctions as Topological Matter, Nat. Commun. 7, 11167 (2016).

[10] E. Eriksson, R.-P. Riwar, M. Houzet, J. S. Meyer, and Y. V. Nazarov, Topological Transconductance Quantization in a Four-Terminal Josephson Junction, Phys. Rev. B 95, 075417 (2017).

[11] H.-y. Xie, M. G. Vavilov, and A. Levchenko, Topological Andreev Bands in Three-Terminal Josephson Junctions, Phys. Rev. B 96, 161406(R) (2017).

[12] X. L. Huang and Y. V. Nazarov, Topology ProtectionUnprotection Transition: Example from Multiterminal Superconducting Nanostructures, Phys. Rev. B 100, 085408 (2019).

[13] E. V. Repin, Y. Chen, and Y. V. Nazarov, Topological Properties of Multiterminal Superconducting Nanostructures: Effect of a Continuous Spectrum, Phys. Rev. B 99, 165414 (2019).

[14] H.-Y. Xie, M. G. Vavilov, and A. Levchenko, Weyl Nodes in Andreev Spectra of Multiterminal Josephson Junctions: Chern Numbers, Conductances, and Supercurrents, Phys. Rev. B 97, 035443 (2018).

[15] J. Erdmanis, Á. Lukács, and Y. V. Nazarov, Weyl Disks: Theoretical Prediction, Phys. Rev. B 98, 241105(R) (2018).

[16] H.-Y. Xie and A. Levchenko, Topological Supercurrents Interaction and Fluctuations in the Multiterminal Josephson Effect, Phys. Rev. B 99, 094519 (2019).

[17] P. Kotetes, M. T. Mercaldo, and M. Cuoco, Synthetic Weyl Points and Chiral Anomaly in Majorana Devices with Nonstandard Andreev-Bound-State Spectra, Phys. Rev. Lett. 123, 126802 (2019).

[18] M. P. Nowak, M. Wimmer, and A. R. Akhmerov, Supercurrent Carried by onequilibrium Quasiparticles in a Multiterminal Josephson Junction, Phys. Rev. B 99, 075416 (2019).

[19] L. P. Gavensky, G. Usaj, and C. A. Balseiro, Topological Phase Diagram of a Three-Terminal Josephson Junction: From the Conventional to the Majorana Regime, Phys. Rev. B 100, 014514 (2019).

[20] V. Kornich, H. S. Barakov, and Y. V. Nazarov, Fine Energy Splitting of Overlapping Andreev Bound States in Multiterminal Superconducting Nanostructures, Phys. Rev. Research 1, 033004 (2019).

[21] M. Houzet and J. S. Meyer, Majorana-Weyl Crossings in Topological Multiterminal Junctions, Phys. Rev. B 100, 014521 (2019).

[22] L. Fu and C. L. Kane, Superconducting Proximity Effect and Majorana Fermions at the Surface of a Topological Insulator, Phys. Rev. Lett. 100, 096407 (2008).

[23] J. Alicea, Y. Oreg, G. Refael, F. Von Oppen, and M. P. A. Fisher, Non-Abelian Statistics and Topological Quantum Information Processing in 1D Wire Networks, Nat. Phys. 7, 412 (2011).

[24] A. Zazunov, R. Egger, M. Alvarado, and A. L. Yeyati, Josephson Effect in Multiterminal Topological Junctions, Phys. Rev. B 96, 024516 (2017).

[25] D. Litinski, M. S. Kesselring, J. Eisert, and F. von Oppen, Combining Topological Hardware and Topological Software: Color-Code Quantum Computing with Topological Superconductor Networks, Phys. Rev. X 7, 031048 (2017).
[26] R. M. Lutchyn, E. P. A. M. Bakkers, L. P. Kouwenhoven, P. Krogstrup, C. M. Marcus, and Y. Oreg, Majorana Zero Modes in Superconductor-Semiconductor Heterostructures, Nat. Rev. Mater. 3, 52 (2018).

[27] M. Trif and P. Simon, Braiding of Majorana Fermions in a Cavity, Phys. Rev. Lett. 122, 236803 (2019).

[28] A. Calzona and B. Trauzettel, Moving Majorana Bound States Between Distinct Helical Edges Across a Quantum Point Contact, Phys. Rev. Research 1, 033212 (2019).

[29] O. Viyuela, S. Vijay, and L. Fu, Scalable Fermionic Error Correction in Majorana Surface Codes, Phys. Rev. B 99, 205114 (2019).

[30] M. Hell, K. Flensberg, and M. Leijnse, Coupling and Braiding Majorana Bound States in Networks Defined in Two-Dimensional Electron Gases with Proximity-Induced Superconductivity, Phys. Rev. B 96, 035444 (2017).

[31] C. Malciu, L. Mazza, and C. Mora, Braiding Majorana Zero Modes Using Quantum Dots, Phys. Rev. B 98, 165426 (2018).

[32] I. C. Fulga, B. van Heck, M. Burrello, and T. Hyart, Effects of Disorder on Coulomb-Assisted Braiding of Majorana Zero Modes, Phys. Rev. B 88, 155435 (2013).

[33] T. Karzig, F. Pientka, G. Refael, and F. von Oppen, Shortcuts to Non-Abelian Braiding, Phys. Rev. B 91, 201102(R) (2015).

[34] B. van Heck, A. R. Akhmerov, F. Hassler, M. Burrello, and C.W.J. Beenakker, Coulomb-Assisted Braiding of Majorana Fermions in a Josephson Junction Array, New J. Phys. 14, 035019 (2012).

[35] T. Karzig, Y. Oreg, G. Refael, and M. H. Freedman, Universal Geometric Path to a Robust Majorana Magic Gate, Phys. Rev. X 6, 031019 (2016).

[36] L. Weithofer, P. Recher, and T. L. Schmidt, Electron Transport in Multiterminal Networks of Majorana Bound States, Phys. Rev. B 90, 205416 (2014).

[37] D. Aasen, M. Hell, R. V. Mishmash, A. Higginbotham, J. Danon, M. Leijnse, T. S. Jespersen, J. A. Folk, C. M. Marcus, K. Flensberg, and J. Alicea, Milestones Toward Majorana-Based Quantum Computing, Phys. Rev. X 6, 031016 (2016).

[38] S. R. Plissard, I. Van Weperen, D. Car, M. A. Verheijen, G. W. G. Immink, J. Kammhuber, L. J. Cornelissen, D. B. Szombati, A. Geresdi, S. M. Frolov et al., Formation and Electronic Properties of InSb Nanocrosses, Nat. Nanotechnol. 8, 859 (2013).

[39] P. Schüffelgen, D. Rosenbach, C. Li, T. W. Schmitt, M. Schleenvoigt, A. R. Jalil, S. Schmitt, J. Kölzer, M. Wang, B. Bennemann et al., Selective Area Growth and Stencil Lithography for In Situ Fabricated Quantum Devices, Nat. Nanotechnol. 14, 825 (2019).

[40] F. Krizek, J. E. Sestoft, P. Aseev, S. Marti-Sanchez, S. Vaitiekėnas, L. Casparis, S. A. Khan, Yu. Liu, T. Stankevič, A. M. Whiticar et al., Field Effect Enhancement in Buffered Quantum Nanowire Networks, Phys. Rev. Mater. 2, 093401 (2018).

[41] P. Aseev, A. Fursina, F. Boekhout, F. Krizek, J. E. Sestoft, F. Borsoi, S. Heedt, G. Wang, L. Binci, S. Martí-Sánchez et al., Selectivity Map for Molecular Beam Epitaxy of Advanced III-V Quantum Nanowire Networks, Nano Lett. 19, 218 (2019). 
[42] S. Vaitiekènas, A. M. Whiticar, M.-T. Deng, F. Krizek, J. E. Sestoft, C. J. Palmstrøm, S. Martí-Sànchez, J. Arbiol, P. Krogstrup, L. Casparis et al., Selective-Area-Grown Semiconductor-Superconductor Hybrids: A Basis for Topological Networks, Phys. Rev. Lett. 121, 147701 (2018).

[43] R. L. M. O. het Veld, D. Xu, V. Schaller, M. A. Verheijen, S. M. E. Peters, J. Jung, C. Tong, Q. Wang, M. W. A. de Moor, Bart Hesselmann et al., In-Plane Selective Area InSb-Al Nanowire Quantum Networks, Commun. Phys. 3, 1 (2020).

[44] A. W. Draelos, M. T. Wei, A. Seredinski, H. Li, Y. Mehta, K. Watanabe, T. Taniguchi, I. V. Borzenets, F. Amet, and G. Finkelstein, Supercurrent Flow in Multiterminal Graphene Josephson Junctions, Nano Lett. 19, 1039 (2019).

[45] A. Freyn, B. Douçot, D. Feinberg, and R. Mélin, Production of Nonlocal Quartets and Phase-Sensitive Entanglement in a Superconducting Beam Splitter, Phys. Rev. Lett. 106, 257005 (2011).

[46] A. H. Pfeffer, J. E. Duvauchelle, H. Courtois, R. Mélin, D. Feinberg, and F. Lefloch, Subgap Structure in the Conductance of a Three-Terminal Josephson Junction, Phys. Rev. B 90, 075401 (2014).

[47] Y. Cohen, Y. Ronen, J.-H. Kang, M. Heiblum, D. Feinberg, R. Mélin, and H. Shtrikman, Nonlocal Supercurrent of Quartets in a Three-Terminal Josephson Junction, Proc. Natl. Acad. Sci. U.S.A. 115, 6991 (2018).

[48] E. Strambini, S. D’Ambrosio, F. Vischi, F. S. Bergeret, Yu. V. Nazarov, and F. Giazotto, The $\omega$-SQUIPT as a Tool to Phase-Engineer Josephson Topological Materials, Nat. Nanotechnol. 11, 1055 (2016).

[49] J. Shabani, M. Kjaergaard, H. J. Suominen, Y. Kim, F. Nichele, K. Pakrouski, T. Stankevic, R. M. Lutchyn, P. Krogstrup, R. Feidenhans et al., Two-Dimensional Epitaxial Superconductor-Semiconductor Heterostructures: A Platform for Topological Superconducting Networks, Phys. Rev. B 93, 155402 (2016).

[50] K. S. Wickramasinghe, W. Mayer, J. Yuan, T. Nguyen, L. Jiao, V. Manucharyan, and J. Shabani, Transport Properties of Near Surface InAs Two-Dimensional Heterostructures, Appl. Phys. Lett. 113, 262104 (2018).

[51] C. W. J. Beenakker, Universal Limit of Critical-Current Fluctuations in Mesoscopic Josephson Junctions, Phys. Rev. Lett. 67, 3836 (1991).

[52] W. Mayer, J. Yuan, K. S. Wickramasinghe, T. Nguyen, M. C. Dartiailh, and J. Shabani, Superconducting Proximity Effect in Epitaxial Al-InAs Heterostructures, Appl. Phys. Lett. 114, 103104 (2019).

[53] G. E. Blonder, M. Tinkham, and T. M. Klapwijk, Transition from Metallic to Tunneling Regimes in Superconducting Microconstrictions: Excess Current, Charge Imbalance, and Supercurrent Conversion, Phys. Rev. B 25, 4515 (1982).
[54] D. Averin and A. Bardas, ac Josephson Effect in a Single Quantum Channel, Phys. Rev. Lett. 75, 1831 (1995).

[55] See Supplemental Material at http://link.aps.org/ supplemental/10.1103/PhysRevX.10.031051 for additional data.

[56] M. H. S. Amin, A. N. Omelyanchouk, and A. M. Zagoskin, Mesoscopic Multiterminal Josephson Structures. I. Effects of Nonlocal Weak Coupling, Low Temp. Phys. 27, 616 (2001).

[57] A. N. Omelyanchouk and M. Zareyan, Ballistic FourTerminal Josephson Junction: Bistable States and Magnetic Flux Transfer, Physica (Amsterdam) 291B, 81 (2000).

[58] G. V. Graziano, J. S. Lee, M. Pendharkar, C. J. Palmstrøm, and V.S. Pribiag, Transport Studies in a Gate-Tunable Three-Terminal Josephson Junction, Phys. Rev. B 101, 054510 (2020).

[59] J.-D. Pillet, C. H. L. Quay, P. Morfin, C. Bena, A. L. Yeyati, and P. Joyez, Andreev Bound States in SupercurrentCarrying Carbon Nanotubes Revealed, Nat. Phys. 6, 965 (2010).

[60] W. Mayer, M. C. Dartiailh, J. Yuan, K. S. Wickramasinghe, A. Matos-Abiague, I. Žutić, and J. Shabani, Phase Signature of Topological Transition in Josephson Junctions, arXiv:1906.01179.

[61] T. Zhou, M. C. Dartiailh, W. Mayer, J. E. Han, A. MatosAbiague, J. Shabani, and I. Žutić, Phase Control of Majorana Bound States in a Topological X Junction, Phys. Rev. Lett. 124, 137001 (2020).

[62] S. Takada, S. Kosaka, and H. Hayakawa, Current Injection Logic Gate with Four Josephson Junctions, Jpn. J. Appl. Phys. 19, 607 (1980).

[63] Z. Qi, H.-Y. Xie, J. Shabani, V. E. Manucharyan, A. Levchenko, and M. G. Vavilov, Controlled-Z Gate for Transmon Qubits Coupled by Semiconductor Junctions, Phys. Rev. B 97, 134518 (2018).

[64] N. Bergeal, R. Vijay, V. E. Manucharyan, I. Siddiqi, R. J. Schoelkopf, S. M. Girvin, and M. H. Devoret, Analog Information Processing at the Quantum Limit with a Josephson Ring Modulator, Nat. Phys. 6, 296 (2010).

[65] N. Bergeal, F. Schackert, M. Metcalfe, R. Vijay, V.E. Manucharyan, L. Frunzio, D. E. Prober, R. J. Schoelkopf, S. M. Girvin, and M. H. Devoret, Phase-Preserving Amplification Near the Quantum Limit with a Josephson Ring Modulator, Nature (London) 465, 64 (2010).

[66] L. B. Ioffe and M. V. Feigelman, Possible Realization of an Ideal Quantum Computer in Josephson Junction Array, Phys. Rev. B 66, 224503 (2002).

Correction: A statement of thanks was inadvertently removed from the Acknowledgments section during production and has been restored. 